\title{
To test, or not to test: time for a MODY calculator?
}

\author{
P. R. Njølstad • A. Molven
}

Received: 13 January 2012 / Accepted: 8 February 2012 / Published online: 2 March 2012

(C) Springer-Verlag 2012

\begin{abstract}
To test, or not to test, that is often the question in diabetes genetics. This is why the paper of Shields et al in the current issue of Diabetologia is so warmly welcomed. MODY is the most common form of monogenic diabetes. Nevertheless, the optimal way of identifying MODY families still poses a challenge both for researchers and clinicians. Hattersley's group in Exeter, UK, have developed an easy-to-use MODY prediction model that can help to identify cases appropriate for genetic testing. By answering eight simple questions on the internet (www.diabetesgenes.org/content/mody-probabilitycalculator), the doctor receives a positive predictive value in return: the probability that the patient has MODY. Thus, the classical binary (yes/no) assessment provided by clinical diagnostic criteria has been substituted by a more rational, quantitative estimate. The model appears to discriminate well between MODY and type 1 and type 2 diabetes when diabetes is diagnosed before the age of 35 years. However, the performance of the MODY probability calculator should now be validated in other settings than where it was developed - and, as always, there is room for some improvements and modifications.
\end{abstract}

\author{
P. R. Njølstad \\ Department of Clinical Medicine, University of Bergen, \\ Bergen, Norway \\ P. R. Njølstad ( $\triangle)$ \\ Department of Pediatrics, Haukeland University Hospital, \\ N-5021 Bergen, Norway \\ e-mail: pal.njolstad@uib.no \\ A. Molven \\ The Gade Institute, University of Bergen, \\ Bergen, Norway \\ A. Molven \\ Department of Pathology, Haukeland University Hospital, \\ Bergen, Norway
}

Keywords Diabetes · Genetic testing · MODY · Monogenic diabetes $\cdot$ Prediction model

Traditionally, MODY was considered if a proband had an age of diabetes onset less than 25 years, there was diabetes in one of his/her parents and the phenotype was characterised by beta cell failure and no obesity [1]. These criteria have been challenged in recent years. Obesity is now frequent in the general population and cannot be used to exclude MODY. Diabetes molecular genetics has provided at least ten forms of MODY, which differ in clinical presentation, including hallmarks such as age of onset after 25 years (mild $H N F 4 A$ mutations), pancreatic exocrine failure (CEL MODY) and renal dysfunction (HNF1B MODY) [2]. Thus, some authors have argued that the term MODY should be considered obsolete and substituted by 'monogenic diabetes' [3]. MODY is, however, still widely used, due both to tradition and to the fact that most cases do indeed fit the classical criteria.

Identification of a MODY mutation is important for correct diagnosis, appropriate genetic counselling, evaluation of prognosis and selection of the best treatment $[2,3]$. The current diagnostic approach when MODY is suspected includes Sanger sequencing of candidate genes, one after another, based on expected clinical and genetic correlations, and dosage analysis where appropriate. Many diagnostic laboratories - including our own - find, however, a mutation in one of the three most common MODY genes in only some $50 \%$ of the probands, with the percentage depending on how stringently the clinical criteria used for inclusion in the screening are set. Most laboratories will then end the screening, although additional genetic investigations are sometimes performed based on a specific phenotype or as part of a research project. The cost of this strategy is 
obviously not trivial because of the number of candidate genes and the iterative screening approach. Although nextgeneration sequencing may change this picture, we are likely to perform Sanger sequencing in the traditional way for several years to come. In the molecular evaluation of MODY, it is very important to exclude type 1 and type 2 diabetes to avoid unnecessary screening. There are some biomarkers associated with either type 1 diabetes or type 2 diabetes, but it can be difficult to define MODY in clinical practice [4].

The article by Shields and colleagues [5] in the current issue of Diabetologia is a significant contribution in this regard. Using logistic regression, the authors have developed a prediction model that discriminates between MODY and type 1 diabetes, and between MODY and type 2 diabetes. The authors used data from 594 individuals with known mutations in the most common genes causing MODY (HNF1A, GCK, HNF4A), 278 individuals with type 1 diabetes and 319 individuals with type 2 diabetes. The model was validated in a set of 350 patients with these three types of diabetes. MODY could best be discriminated from type 1 diabetes by lower $\mathrm{HbA}_{1 \mathrm{c}}$, parent with diabetes, being female and older age at diagnosis, while MODY was discriminated from type 2 diabetes by lower BMI, younger age at diagnosis, being female, lower $\mathrm{HbA}_{1 \mathrm{c}}$, parent with diabetes, and not being treated with tablets or insulin. The model showed very good discrimination and a low rate of misclassification, and performed well on the external data set. Using optimal cut-offs, the prediction model improved the sensitivity from $72 \%$ to $91 \%$ and the specificity from $91 \%$ to $94 \%$ for identifying MODY compared with the standard criteria.
A very attractive feature of the proposed model is its ease of use (Fig. 1). By punching eight straightforward features of the diabetes patient into the MODY calculator on the website (www.diabetesgenes.org/content/modyprobability-calculator), a positive predictive value, i.e. the probability that the patient has MODY, is calculated. An important point is that the physician will receive a quantitative estimate for the diagnosis rather than the conventional yes/no evaluation provided by clinical diagnostic criteria.

Age at diagnosis and family history are crucial elements in a predictive model of genetic disease, including MODY. Although most physicians are aware that inaccurate family history is a frequently encountered problem when taking a case history, few try to contact other relatives to verify reported family cases. Inaccurate family history may distort the prediction, which again may influence clinical management and the decision of whom to test genetically. Typically, misreported age at diagnosis causes less distortion in the prediction than a misreported disease diagnosis [6]. Moreover, the amount of distortion of prediction usually decreases with age of a relative, but also depends on the relationship to the proband and outcomes in other relatives. Hence, a misreported diagnosis in a second degree relative causes greater distortion of the prediction if the first degree relative is affected rather than unaffected [6]. In the questionnaire of Shields et al [5], the issue regarding a precise family history has been replaced by the simple question: Parent with diabetes-yes or no! It will be interesting to see whether this simplification makes the predictive model even more robust.

The study populations in the article by Shields et al [5] comprised individuals mainly referred from physicians for
Fig. 1 The MODY calculator. By answering eight simple questions on the internet, the probability (positive predictive value) of a diabetes patient having MODY is returned. OHA, oral hypoglycaemic agent. Adapted from www. diabetesgenes.org with the permission of A. T. Hattersley
1. Age at diagnosis

2. Sex

\section{Currently treated with insulin or OHA?}

4. Time to insulin treatment (if currently treated with insulin)

5. BMI

6. $\mathrm{HbA} 1 \mathrm{c}$

7. Current age

8. Parent affected with diabetes? $\bigcirc$ Yes $\bigcirc$ No years

Male O Female

Yes O No

Not currently treated with insulin

W Within 6 months of diagnosis

Over 6 months after diagnosis

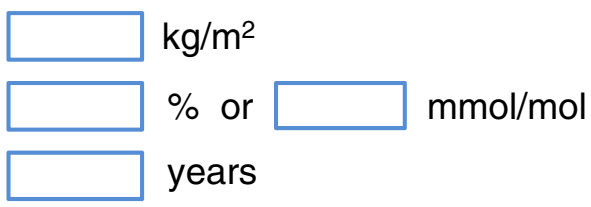


genetic testing. Thus, it may be argued that the patients' family histories are not as complete as would be desirable. On the other hand, an advantage is that the proposed clinical model may fit the need of general practitioners rather than that of expert medical geneticists or diabetologists working in clinics, where complementary and better tools may be available.

Although a mutation in any of the genes HNF1A, HNF4A and $G C K$ is present in more than three of four individuals with genetically confirmed MODY, a limitation of the MODY calculator is that presence of extra-pancreatic features is ignored. If this was included, the model would probably have picked up, for example, HNF1B MODY, which is a systemic disease including renal cysts, renal failure, genital abnormalities, abnormal liver function and dorsal pancreas aplasia [7].

Another important question is whether biomarkers should be part of the prediction model. The advantage of clinical characteristics alone is that they are routinely available to all doctors caring for patients with diabetes. In the model presented, clinical characteristics discriminated MODY well on their own, without the need for data from further biomarkers, which many clinicians will not have available. To incorporate biomarkers is, however, a logical progression of the prediction model. The search for and use of relevant biomarkers in diabetes has attracted increasing attention: the inclusion of selected markers, for instance pancreatic autoantibodies, C-peptides, HDL-cholesterol and high-sensitivity $\mathrm{C}$-reactive protein (hsCRP), in the model will probably enhance the its value [8].

The prediction model of Shields et al [5] is based solely on white people and may not be applicable to other populations. A study has shown that the prediction power of risk prediction models widely used for $B R C A 1$ and $B R C A 2$ mutations had lower sensitivity, specificity and positivepredictive value in a Malayan than in a white population [9]. Hence, the MODY calculator needs to be validated in non-white populations as well.

When testing for monogenic disease by Sanger sequencing, high sensitivity is inexorably associated with high costs if there are many candidate genes. Using a prediction model to lower costs without missing mutation carriers is essential. The cost-effectiveness is determined by the cut-off values used, with lower cut-off values resulting in higher costs per extra detected mutation. The optimal threshold in terms of cost-effectiveness is not known and may depend on the specific healthcare setting. In addition, the nature of the mutation detected is crucial. One might, for example, accept a high cost per detected mutation when a positive test result might change treatment, for instance when detecting a pathogenic mutation in HNF1A that would indicate an attempted switch from insulin to sulfonylurea [10].
How should the prediction model be used to select cases for genetic screening? Due to the selection of age at diagnosis in the materials used in the study, the model would only be applicative in patients diagnosed with diabetes aged between 1 and 35 years. In cases where a patient is insulintreated soon after diagnosis, a probability greater than $10 \%$ might represent a suitable level where further testing for C-peptide and islet autoantibodies may be considered justified. Presence of C-peptide and absence of pancreatic autoantibodies would then suggest genetic testing. In cases where patients are not insulin-treated soon after diagnosis, a probability of more than $25 \%$ would be a reasonable level at which genetic testing may be offered.

Who is going to use the MODY calculator? We regard it as a valuable research tool that can be employed to prioritise patient samples for genetic testing. For the busy clinician seeing a new patient with diabetes, the calculator will not on its own settle the issue of whether or not to test. A weighted decision based on the probability of MODY, any extrapancreatic findings, the costs of the tests, and the impact of a genetic diagnosis on prognosis and treatment should always be made.

Acknowledgements We acknowledge the many discussions with colleagues in the field.

Funding We received financial support from the University of Bergen, the Research Council of Norway and Helse Vest.

Contribution statement PRN and AM were responsible for the interpretation of data and literature, wrote and revised the article, and gave approval of the final version to be published.

Duality of interest P. R. Njølstad has received research support from Novo Nordisk. He is President of the EASD Study Group for the Genetics of Diabetes (SGGD). The views expressed in this commentary are not necessarily those held by the EASD-SGGD.

\section{References}

1. Tattersall FSS (1975) A difference between the inheritance of classical juvenile-onset and maturity-onset type diabetes of young people. Diabetes 24:44-53

2. Molven A, Njølstad PR (2011) Role of molecular genetics in transforming diagnosis of diabetes mellitus. Expert Rev Mol Diagn 11:313-320

3. Murphy R, Ellard S, Hattersley AT (2008) Clinical implications of a molecular genetic classification of monogenic beta-cell diabetes. Nat Clin Pract Endocrinol Metab 4:200-213

4. Shields BM, Hicks S, Shepherd MH, Colclough K, Hattersley AT, Ellard S (2010) Maturity-onset diabetes of the young (MODY): how many cases are we missing? Diabetologia 53:2504-2508

5. Shields BM, McDonald TJ, Ellard S, Campbell MJ, Hyde C, Hattersley AT (2012) The development and validation of a clinical 
prediction model to determine the probability of MODY in patients with young-onset diabetes. Diabetologia. doi:10.1007/s00125011-2418-8

6. Katki HA (2006) Effect of misreported family history on Mendelian mutation prediction models. Biometrics 62:478-487

7. Haldorsen IS, Ræder H, Vesterhus M, Molven A, Njølstad PR (2011) The role of pancreatic imaging in monogenic diabetes mellitus. Nat Rev Endocrinol 8:148-159

8. Thanabalasingham G, Shah N, Vaxillaire M et al (2011) A large multi-centre European study validates high-sensitivity C-reactive protein (hsCRP) as a clinical biomarker for the diagnosis of diabetes subtypes. Diabetologia 54:2801-2810

9. Thirthagiri E, Lee SY, Kang P et al (2008) Evaluation of BRCA1 and BRCA2 mutations and risk-prediction models in a typical Asian country (Malaysia) with a relatively low incidence of breast cancer. Breast Cancer Res 10:R59

10. Shepherd M, Pearson ER, Houghton J, Salt G, Ellard S, Hattersley AT (2003) No deterioration in glycemic control in HNF-1alpha maturity-onset diabetes of the young following transfer from longterm insulin to sulphonylureas. Diabetes Care 26:3191-3192 